\title{
Evaluation of cellular and molecular and anti- apoptotic effects of lipid nanoparticle-containing Artemisia extract in ovarian cancer cells
}

\section{Farnaz Aboutalebi}

Azad University: Islamic Azad University

Maryam Teimouri ( $\square$ teimourimaryam93@gmail.com )

Azad University: Islamic Azad University https://orcid.org/0000-0002-6609-5415

\section{Mehdi Pooladi}

Azad University: Islamic Azad University

\section{Research Article}

Keywords: Artemisia, ovarian cancer, apoptotic, nanoparticle

Posted Date: September 29th, 2021

DOI: https://doi.org/10.21203/rs.3.rs-946667/v1

License: (c) (i) This work is licensed under a Creative Commons Attribution 4.0 International License.

Read Full License 


\section{Abstract}

Ovarian cancer is the fifth reason for the woman's death. When cells get malignant changes in one or both ovaries, ovarian cancer gets formed. This kind of cancer when it is under control, it influenced the fertility of the patient. Artemisia Aucheri Boiss is used for most of the diseases to be healed. Due to the importance of ovarian cancer and the potential of anti-Apoptotic of Artemisia Aucheri Boiss, in this research the extraction of this plant in lipid nanoparticles with the target of cellular and molecular effects and anti Apoptotic loaded on the ovarian cancer cells. In this examination, first nanoparticles containing Artemisia extraction were synthesized by homogenizer plus sonication and subsequently their physical and chemical properties were determined like (size and shape of particles, description of particles, zeta potential, rate of efficiency of essential oil. Then the anti Apoptotic effect of lipid nanoparticle-containing Artemisia extraction in ovarian cancer cells (SKOV-3 line) was determined by MTT assay and flow cytometry analysis.Results of the cellular effects of lipid nanoparticle-containing extraction of the plant on the ovarian cancer cells show that the increase of viscosity of lipid nanoparticle containing the essence of this plant led to disease in the viability of ovarian cancer cells compared to pure essence. It seem that lipid nanoparticles appear to activate the apoptotic pathway in ovarian cancer cells.

\section{Introduction}

Cancer is one of the causes of death in more than one-third of the world population and caused the death of more than $20 \%$ of all people around the world $[1,2]$. Ovarian cancer is the second most common cancer between women and causes the deaths of malignant genital cancer. Ovarian cancer is one of the nine most common cancers and is one of the five major reasons for cancer death between women that is easily detectable and treatable in early stages but if postponed, treatment will be difficult and at some points unsuccessful [3-5].

The most common form of ovarian cancer is epithelial ovarian cancer which is without any symptom and more than $70 \%$ of patients have diagnosed in the advanced level of disease that the first line of standard treatment consisted of surgical tumor determination and combination chemotherapy $[6,7]$.

Resistance to chemotherapy creates a barrier to ovarian cancer treatment. In order to overcome the need to find more effective or complementary therapies with fewer side effects, longer life spans are essential $[8,9]$.

Pieces of evidence and the World Health Organization (WHO) show that about $70 \%$ of the population around the world prefer traditional herbal remedies to treat their ailments. In Iran, the usage of alternative medicine has increased dramatically in the past decade [10]. So that about $60 \%$ of all anti-cancer agents come from medicational plants and other natural sources [11]. However many plants still have anticancer potential that has not yet been investigated yet. Therefore the suggested solution for the harmful effects of chemical drugs, into the use or substitution of herbal remedies. One of these herbal remedies is Artemisia which belongs to Asteraceae family [12]. Many types of this family have shown healing 
properties due to the presence of metabolites such as flavonoids, coumarins, steroids, polyethylene, mono and ciscoitpens, and lactonsuzoitropen [13].

Encapsulating herbal essence is one of the most effective ways to increase the solubility and stability of essential oils under adverse environmental conditions and to control its active ingredients [14].

The development of nanosize formulations of bioactive compounds due to their small size has more advantages than other encapsulation systems. These systems have higher intracellular uptake and function because of their size. Due to the fatty nature of herbal essence, Solid lipid nanoparticles (SLN) because of biocompatibility, low toxicity and good degradability, with the ability to encapsulate hydrophobic biological compounds can be used as a very beneficial option for carrying plants extraction $[8,15]$.

According to the importance of ovarian cancer and the toxic effect of some Artemisia types on cancer cells, in this study, the extraction of the plant was used. During different formulations using surfactant and different essential fats are loaded into AR.SLN using a homogenizer. Chemical- physical properties of the extraction on nanoparticles and the rate of release of the extraction were studied by UV-vis, Zetasizer and dialysis bag. Finally, the cytolytic activity of loaded extraction in nanoparticles compared to pure extraction on the SKOV-3 cell line compared to HEK-293 normal cell line was performed by quantitative tetracycline evaluated by MTT assay based on the colorimetric method and also flow cytometry.

\section{Methods}

Collection and identification of constituents of Artemisia (Wild type) For this research, the branches of Artemisia (With the scientific name Artemisia Aucheri Boiss) from the geographical area of Kerman province in Iran. The drying step of the plant was carried out, to begin with, removal of waste material from direct sunlight, then the dried plant was milled and the essence was collected by the Clevenger apparatus.

\section{UV-VIS Spectral studies}

To determine the maximum UV-Vis (SPECORD 210, Analytik jena, Germany) wavelength of the essence, the UV-Vis device was absorbed in the range of 200 to $400 \mathrm{~nm}$. For this purpose, the stock was prepared $100 \mu \mathrm{l}$ viscosity at about $0.9 \mathrm{~g} / \mu \mathrm{l}$ density. The volumetric phase was carried out by ethanol $99.8 \%$ in 10 cc Balloon. The calibration curve was drawn according to the standard and the prepared viscosity.

\section{Method of preparation of SLN containing Artemisia}

Melt high-pressure homogenizer method was used to make nanoparticle. ${ }^{12}$ For this study, two types of lipophilic surfactant (solid span 60, liquid span 80) and one hydrofoil surfactant and five types of solid lipid (Stearic acid, palmitic acid, cholesterol, bismuth and glyceryl monostearate) were selected. In this case, the lipid phase containing the lipophilic surfactant of the essence and the fat reached $a 0^{\circ} \mathrm{C}$ is 
added to the aqueous phase containing the lipid phase isothermal surfactant under milk homogenizers. The initial emission is then homogenized at a pressure 250 times in three consecutive cycles by a highpressure homogenizer. After all, the sample is cooled to ambient temperature to form nanoparticles. Placebo was also prepared according to the above method.

\section{Physical and chemical characterization of SLN containing Artemisia}

Determination of particle size distribution and specific Zeta potential for drug stability testing by Malvern Zetasizer (Nano ZS) at room temperature and $90^{\circ}$ angle. To evaluate the above parameters, the device was calibrated based on aqueous phase (RI: 1.3) and then synthesized drug nanoparticles were passed through syringe filter with size $0.22 \mathrm{~mm}$. The morphology of the nanoparticles containing the essence was imaged by a TEM electron microscope.

\section{Determination of the percentage of essence loaded and the rate of essence release}

Percentage of loaded essence was calculated according to formula 1 membrane diffusion and dialysis bag with the size of 12000 Dalton were used for the calculation of essence release for 24 hours. $m_{e}$ : the amount of drug confined; $\mathrm{m}_{0}$ : primary drug level.

\section{$E E(\%)=\frac{m e}{m 0} \times 100$}

\section{Cell culture and grouping}

Human ovarian cancer (ATCC Code: HTB-77) cell line (SKOV-3) as well as normal cell line (HEK293) were performed according to the protocol provided by Pasteur Institute of Iran cell bank. Accordingly, cells were cultured in PRMI medium supplement with $10 \%$ fetal bovine serum as well as penicillin in $\mathrm{CO}_{2}$ incubate at $35^{\circ} \mathrm{C}$. Two cell lines were visualized for 24 hours with different viscosity of nanoparticles and analyzed.

\section{SKOV-3 cell lines}

The first group (SKOV-3 control); Nano treatment was given without treatment.

The second group (SKOV-3 $+25 \mu$ l nano drug-containing essence)

The third group (SKOV-3 cells $+50{ }^{\circ} \mu \mathrm{l}$ nano drug-containing essence)

The fourth group (SKOV-3 cells $+100 \mu$ l nano drug-containing essence)

\section{HEK-293 cell lines}

The first group (HEK-293 control); Nano treatment was given without treatment

The Second group (HEK-293 $+25 \mu \mathrm{g} / \mathrm{ml}$ nano containing essence) 
The Third group (HEK-293 + $50 \mu \mathrm{g} / \mathrm{ml}$ nano essence)

The fourth group (HEK-293 + $100 \mu \mathrm{g} / \mathrm{ml}$ nano containing essence)

\section{Determination of IC50 values MTT assay}

In these tests, the data was exposed to different essence and the number of dead cells. In this case, yellow Tetrazolium and the formation of Formazon insoluble violet crystals with MTT assay method were used. In this method, 24 hours after cell culture in a designed viscosity, the medium culture was replaced and the cells were cultured for 3 hours with Tetrazolium then the medium was combined with dimethyl sulfoxide microliters barrier and the rate and intensity of absorption were read using a wavelength Ads ELISA Readers (AsysHitchech, Austria).

\section{Cell Viability $(\%)=\frac{\mathrm{A}(\text { treated })}{\mathrm{A}(\text { Control })} \times 100$}

\section{Flow Cytometry Test}

Flow Cytometry was used to measure cell growth inhibition. The cells were trypsinized after the respective treatments, then centrifuged at 1200 and re-suspended in five $\mathrm{ml}$ of phosphate buffer. After centrifugation, added $1 \mathrm{ml}$ of the kit buffer and after severe pipetting, added five microliters of his annexin and incubated in the dark for minutes. In the end, add $5 \mu \mathrm{l}$ of propidium iodide solution and analyzed by flow cytometry.

\section{Extraction, RNA measurement of quantity and quality}

In order to qualitatively evaluate, the $2 \mu \mathrm{l}$ f sample by horizontal agarose gel electrophoresis $1 \%$ was investigated. The $18 \mathrm{~s}$ and $28 \mathrm{~s}$ bands associated with ribosomal RNAs are considered as indicators of extracted RNA quality.

\section{cDNA synthesis}

The kits of Vivantis Malasia and thermocycler were used for cDNA synthesis reactions. The sequencing program is shown in figure 1 . The RT-PCR reaction was performed to analyze the accuracy of DNA synthesis. The polymerase chain reaction of FxR primers genes like BAX, BCL2, GAPDH with GTP Master MIX, 2X was used by the Pishgaman gene transfer company.

\section{Data analysis method}

SPSS software was used statistical for analysis of nanoparticle tests and the significance of the results was measured in $\mathrm{P}<0.05$. After performing a Real-Time PCR reaction, the device data were calculated using SPSS software and Turkish test and one way ANOVA in the respective formulas. 


\section{Results}

\section{Physical and chemical characterization analysis of SLN}

By UV-VIS, the maximum absorbance of the essence is $274 \mathrm{~nm}$ for which the calibration curve of essence is plotted, followed by the mean absorbance and standard deviation at $254 \mathrm{~nm}$ in figure 2 .

The first indication of the production of lipid nanoparticle has been the discoloration of the synthesized compound. The color change was obtained after $24 \mathrm{~h}$ of incubation. The DLS study showed that the average size of lipid nanoparticles contained the essence of Artemisia $112.8 \mathrm{~nm}$, as shown in the diagram (figure 3). We also report the Zeta potential for the synthesized compound (figure 4). In the continuous TEM electron microscope image to confirm (figure 5) this image shows that the lipid nanoparticles containing a synthetic spherical essence and are well dispersed. The bright background around the spherical nanoparticle is solvent related. This contrast is due to differences in the viscosity of the synthesized nanoparticle and used solvent.

\section{Drug release}

According to the diagram, it was observed that the lipid phase of glycerol nano-stearate and surfactant span 80 had a higher release than the lipid phase glycerol monostearate and surfactant span 60 . RA-SLN formulation releases $47 \%$ of the essence in the first 2 hours and releases $54 \%$ of the essence in the first 24 hours. Whereas in RA-SLN4 formulation $39 \%$ was released in the first 2 hours and in the $50 \%$ in the 24 hours. Also, it is cleared that the release of the essence is less than formulations. Therefore, lipid nanocarriers increased the rate of essence release compared to pure essence.

Table. 1 Physicochemical properties of Nanoparticles containing Artemisia extraction ( weight percent/ weight). The formulation was repeated three times and the table was reported with standard deviation. AR: Artemisia (Col: cholesterol; BW: bees wax; GMS; glycerol monostearate; SA: stearic acid; PA: palmitic acid; T 20: tween 20; Sp60: span60) PDI: polydispersity index; ZP: zeta potential; EE: entrapment efficie 


\begin{tabular}{|c|c|c|c|c|c|}
\hline Formula & Size (nm) & PDI & $\mathrm{ZP}(\mathrm{mv})$ & $\mathrm{EE} \%$ & \\
\hline AR-SLN1 & $329.73 \pm 21.64$ & $0.291 \pm 0.015$ & $-14.96 \pm 0.75$ & $45.60 \pm 1.52$ & \\
\hline AR-SLN2 & $352.8 \pm 13.95$ & $0.335 \pm 0.061$ & $-9.86 \pm 2.034$ & $51.12 \pm 0.62$ & \\
\hline AR-SLN3 & $156.97 \pm 7.15$ & $0.388 \pm 0.004$ & $-27.67 \pm 1.89$ & $59.02 \pm 0.74$ & \\
\hline AR-SLN4 & $176.33 \pm 19.44$ & $0.424 \pm 0.009$ & $-24.93 \pm 0.55$ & $58.90 \pm 0.20$ & \\
\hline AR-SLN5 & $268.2 \pm 7.84$ & $0.230 \pm 0.009$ & $-19.3 \pm 1.04$ & $54.46 \pm 3.93$ & \\
\hline AR-SLN6 & $392.13 \pm 28.79$ & $0.274 \pm 0.06$ & $-3.18 \pm 1.11$ & $59.47 \pm 0.47$ & \\
\hline AR-SLN7 & $198.53 \pm 22.55$ & $0.322 \pm 0.045$ & $-21.8 \pm 2.43$ & $56.96 \pm 2.47$ & Analysis of clinical findings \\
\hline AR-SLN8 & $303.03 \pm 8.25$ & $0.259 \pm 0.018$ & $-11.37 \pm 5.74$ & $52.69 \pm 0.93$ & $\begin{array}{l}\text { (cellular inhibition and } \\
\text { apoptosis rate) }\end{array}$ \\
\hline
\end{tabular}

Figure 7A and $B$ shows the MTT test at the viscosity of $0,1,25,50,100 \mathrm{mM}$ of Artemisia plant extract after 24 hours. As can be seen by increasing the essence viscosity, the viability of SKOV-3 and HEK-293 cell lines decreased, and the results showed that the lowest viability was observed in SKOV-3 cancer cells at $100 \mathrm{mM}$ and in normal cells at $100 \mathrm{mM}$ essence was obtained. The highest viability of SKOV-3 and HEK-293 cell lines was achieved at 0 and $1 \mathrm{mM}$ essence viscosity. According to the results, $50 \%$ of cell death was reported for SKOV-3, cancer cell lines, at 100mM cellular viscosity. For the HEK-293, IC50 was reported at $100 \mathrm{mM}$ viscosity. In the following IC50`s viscosity of Artemisia essence as well as nanoparticles containing Artemisia extraction significantly increased apoptosis compared to the control group $(\mathrm{P}<0.001)$ (figure $7 \mathrm{C}$ ) the results also showed an increase in apoptosis in SKOV-3 carcinoma cells due to nanoparticles containing Artemisia extraction compound to Artemisia extraction treatment. In other words, nanoparticle treatment with Artemisia plant extract significantly increased apoptosis compared to the control group. $(P<0.001)$ ( figure 7D).

This suggested that nanoparticles containing Artemisia extraction caused more apoptosis in SKOV-3 and HEK-293 line cells than Artemisia extraction group. It can be concluded that one of the anti-cancer and cytotoxic mechanisms of nanoparticles containing Artemisia is to increase the apoptosis of cancer cells. It should be noticed that in the percentage of apoptotic cells in SKOV-3 and HEK-293 line cells were significantly increased compared to the control group.

\section{Molecular results analysis}

The results of the evaluation of RNA molecules extracted by the spectrophotometer, together showed that the OD $260 / 280$ ratio of all the extracted samples was about 1.92 to 1.97 which indicates a good quality of extraction. Also, evaluation of the extracted RNA molecule by gel electrophoresis method about $1 \mu \mathrm{g}$ of the sample was investigated by horizontal electrophoresis on $1 \%$ agarose gel. The $18 \mathrm{~s}$ and $28 \mathrm{~s}$ bands associated with the ribosomal RNA were considered as the extracted RNA quality index that the results showed that the purification of the RNA molecule was good in quality for continuing this procedure. 
DNA synthesis was performed for a polymerase chain reaction, using BAX and BCL2 and actin gene FxR primers. After the end of the replication reaction, $5 \mu \mathrm{l}$ of the sample was re-suspended in $2 \%$ agarose gel to evaluate the quality of the synthesized cDNA. The results of cDNA synthesis analysis showed that high quality synthesized is suitable for Real-Time PCR reactions (figure 10).

In order to perform Polymerase reaction in Real-Time PCR device, first the viscosity of forward and Reverse primers were optimized. Table 2 shows the viscosity used to optimize the PCR conditionsoptimal primers values were obtained after the Polymerase chain reaction for control and $\beta$-actin, Bax and BCL2 genes.

The results of the melting curve analysis of Bax, $\beta$-actin, BCL2 genes are shown in figure 11. It should be noted that the unit melting curve indicates the absence of a non-specific product.

After Real-Time reaction, the data of the device in the relevant formulas were calculated and the values were analyzed by SPSS software, Turkey and one-way ANOVA tests. The expression level of Bax and Bcl2 genes is increased compared to the internal reference gene of GAPDH. As the results of the expression analysis show, the expression of the BAX gene increased about 2.6 compared to the $\beta$-actin internal control gene, however, BCL2 gene expression increased about 1.7 compared to internal control. Therefore, it can be deduced that lipid nanoparticle-containing essence increase the expression of BAX and BCL2 genes which is one of the important genes of the apoptosis pathway. In fact, lipid nanoparticles containing essence activated the apoptosis pathway in ovarian cancer cells and subsequently caused tumor growth to be stopped.

\section{Discussion}

Due to the importance of ovarian cancer and the anti-oxidant potential of Artemisia, this study is aimed to investigate the cellular, molecular and anti-apoptotic effects of the essence on lipid nanoparticles in ovarian cancer cells. The major constituents of lipid nanoparticles include comprehensive lipid surfactants and water. The physicochemical and stability properties of solid lipid nanoparticles depend on the properties of the active ingredient and its compounds. Proper choice of fats, surfactants, their compounds, and their amounts can affect particle size, long term storage stability, a drug loading, and release behaviors. This means that each drug requires a special formulation for SLN [16, 17], which by modifying some of these factors we have found the most appropriates basis for importing Artemisia extraction. In the previous study the presence of alpha-pinene, Beta-pinene, D germacrene, and limonene, in Artemisia has been inhibiting the growth of human breast, liver, and melanoma cancer cells. Jupatolin is also one of the inductors of apoptosis in HL-60 cells and gastric cancer [18].

SLNs are made of one or a mixture of lipids that are solid at ambient temperature and body temperature. The type and amount of lipids affect the particle size of the nanoparticles. Each lipid has several chemical constituents in its structure that can be different in each structure, which in turn affects the important parameters of the nanoparticles [19]. The viscosity amount of lipid used nanoparticles can be different from $2-8 \%$ the amount of lipid used depends on the nanoparticle manufacturing technique. 
Studies have shown that an increase in lipid viscosity is associated with an increase in drug entry into the nanoparticles. On the other hand, an increase in lipid content will increase the particle size [20]. In this study, lipid content was applied 4 times as extraction and due to the low amount of extracted essence of the plant, only 4 formulation was prepared using two types of lipids and two types of surfactants. Then the differences of lipid and surfactant type as the influencing factor on the prepared nanoparticle parameters such as particle size and distribution were investigated. The results of a study by Akrout and his colleagues showed that the methanolic extraction of various parts of wild Artemisia has cytotoxic effects on MFC7 cells [21]. In a similar study, Gordanian and his colleagues reported that toxicity in the aerial parts of Artemisia was higher than in other organs. They have reported the reason for the accumulation of anti-oxidant substances in the shoots, which is the reason why we have also the shoots of Artemisia in our research [22, 23].

The type and amount of surfactant is the most important factor affecting the quality of the nanoparticles. unfavorable and low use of surfactants increases particle size and does not even create nanoparticle. Depending on the lipid viscosity in the formulation the amount of surfactant is used from $0.5-5 \%$. Different surfactants have different distribution on the surface of the nanoparticles, which are better than using several surfactants to achieve optimum particle size and stability [24]. Numerous researches have suggested that the usage of surfactant with HLB lipophilic side by side with surfactant with Hydrophilic HLB increases the Zeta potential and thereby increases the stability. For this reason, in this study, the combination of two surfactants Twin 80 with HLB Hydrophilic and Span 60 with 4.3 HLB was used. On the other hand, studies have shown that by increasing the surfactant with Hypophilic HLB, the particle size increases ${ }^{21}$ so in this study, we think of the ratio of Twin to span 1:20.

One of the benefits of Real-Time PCR is the melting curve drawing that was completed after the PCR process. The DNA melting temperature is a specific parameter for this molecular and depends on the DNA structure and on factors such as length and number of nuclei, protein concentration, ambient salt content, and GC percentage. Since SYBR cannot distinguish between different products, the melt curve can be used to determine the variety of products in the PCR process. After the PCR is completed, the machine is able to draw the melting diagram of each sample. This is done by measuring the fluorescence changes at different temperatures [2, 25].

The results of the cellular effects of lipid nanoparticle-containing essence on ovarian cancer cells showed that increasing the viscosity of lipid nanoparticles containing Artemisia causes the reduction of survival of Hella cells. Our results are in agreement with the findings of Asghari et al (2012) which in their study on the evaluation of the effect of methanolic extraction of Artemisia showed that it possesses antioxidant and cell lethal properties and has potential for use in cancer treatment. They showed that an increase in the viscosity of extraction of Artemisia reduced the viability of cancer cells, in a dosedependent manner [26]. Similarly, Gharehmatrossian et al (2012) showed that methanol extraction has the ability to inhibit cell growth in a concentration-dependent manner [27].

\section{Conclusion}


Overall, the results of our study showed that lipid nanoparticles containing the extraction of Artemisia plant activated the Apoptosic pathway in ovarian cancer cells and subsequently caused tumor growth arrest.

\section{Declarations}

\section{Ethical Approval}

Not applicable

\section{Consent to Participate}

I confirm that the final manuscript has been seen and approved by all the authors. The undersigned author transfers all copyright ownership of the manuscript to The Internal Journal of Applied Biochemistry and Biotechnology in the event the work is published.

\section{Consent to Publish}

We hope that you will find our manuscript acceptable for publication in the above Journal.

\section{Authors Contributions}

M Pooladi and, M Teimouri designed the study, dathcred and analyzed the data and wrote the paper. $\mathrm{F}$ Aboutalebi, and M Pooladi contributed to study desigh.

\section{Funding}

This research was funded by Islamic Azad University, Iran.

\section{Competing Interests}

The authors declare no conflict of interest

\section{Availability of data and materials}

Not applicable

\section{References}

1. Pooladi M, Abad SK, Hashemi M (2014) Proteomics analysis of human brain glial cell proteome by 2D gel. Indian journal of cancer 51:159

2. Teimouri M, Pooladi M (2021) Anti-Angiogenic and Anti-Proliferative Effects of Physalis Alkekengi Hydroalcholic Extract on Breast Cancer in Mice. Journal of Fasa University of Medical Sciences 10:3684-3691 
3. Fleet JC, DeSmet M, Johnson R, Li Y (2012) Vitamin D and cancer: a review of molecular mechanisms. Biochem J 441:61-76

4. Ebrahimi M, Teimouri M, Pooladi M (2021) The synergistic anticancer traits of graphene oxide plus doxorubicin against BT474 and MCF7 breast cancer stem cells in vitro. Appl Biochem Biotechnol 2021:1-6

5. Teimouri M, Odoumizadeh M (2021) Cytotoxicity of Artemisia Vulgaris Essential oil Encapsulated in SLN on Breast Cancer Cell Line (MCF7). Archives of Advances in Biosciences 12:11-26

6. Moore RG, Brown AK, Miller MC, Skates S, Allard WJ, Verch T et al (2008) The use of multiple novel tumor biomarkers for the detection of ovarian carcinoma in patients with a pelvic mass. Gynecol Oncol 108:402-408

7. Bookman MA (2012) First-line chemotherapy in epithelial ovarian cancer. Clin Obstet Gynecol 55:96113

8. Ehsanfar P, Teimouri M, Pooladi M (2020) Investigating Characterizations and Antifungal Effects of Solid Lipid Nanoparticles (SLNs) Loaded with Essential Oil of Citrus Aurantifolia on Isolated Malassezia Strains. Archives of Advances in Biosciences 11:43-55

9. Siegel RL, Miller KD, Jemal A (2019) Cancer statistics, 2019. Cancer J Clin 69:7-34

10. Wang CZ, Calway T, Yuan CS (2012) Herbal medicines as adjuvants for cancer therapeutics. Am J Chin Med 40:657-669

11. Salimifar M, Fatehi-Hassanabad Z, Fatehi M (2013) A review on natural products for controlling type 2 diabetes with an emphasis on their mechanisms of actions. Curr Diabetes Rev 9:402-411

12. Jafariparizi M, Afsharzadeh S, Akkafi HR, Abbasi S (2017) Floristic study of Artemisia aucheri Boiss. rangelands in Isfahan Province, Iran. Nova Biologica Reperta 4:236-245

13. Bora KS, Sharma A (2011) The genus Artemisia: a comprehensive review. Pharm Biol 49:101-109

14. Lai F, Sinico C, De Logu A, Zaru M, Müller RH, Fadda AM (2007) SLN as a topical delivery system for Artemisia arborescens essential oil: in vitro antiviral activity and skin permeation study. Int $\mathrm{J}$ Nanomed 2:419

15. Müller RH, Mäder K, Gohla S (2000) Solid lipid nanoparticles (SLN) for controlled drug delivery-a review of the state of the art. European journal of pharmaceutics biopharmaceutics 50:161-177

16. Mukherjee S, Ray S, Thakur RS (2009) Solid lipid nanoparticles: a modern formulation approach in drug delivery system. Indian journal of pharmaceutical sciences 71:349

17. Teoh DG, Secord AA (2011) Antiangiogenic therapies in epithelial ovarian cancer. Cancer Control 18:31-43

18. Raha S, Robinson BH (2000) Mitochondria, oxygen free radicals, disease and ageing. Trends Biochem Sci 25:502-508

19. Bobrowski K (2005) Free radicals in chemistry, biology and medicine: contribution of radiation chemistry. Nukleonika 50:67-76 
20. Lingayat VJ, Zarekar NS, Shendge RS (2017) Solid lipid nanoparticles: a review. Nanoscience Nanotechnology Research 2:67-72

21. Akrout A, Chemli R, Chreif I, Hammami M (2001) Analysis of the essential oil of Artemisia campestris L. Flavour Fragr J 16:337-339

22. Gordanian B, Behbahani M, Carapetian J, Fazilati M (2014) In vitro evaluation of cytotoxic activity of flower, leaf, stem and root extracts of five Artemisia species. Research in Pharmaceutical Sciences 9:91

23. Emami SA, Rabe SZ, Ahi A, Mahmoudi M, Tabasi N (2009) Study the cytotoxic and pro-apoptotic activity of Artemisia annua extracts. Pharmacologyonline 3:1062-1069

24. Mahmoudi M, Rabe SZ, Ahi A, Emami SA (2009) Evaluation of the cytotoxic activity of different Artemisia khorassanica samples on cancer cell lines. Pharmacol online 2:778-786

25. Wong ML, Medrano JF (2005) Real-time PCR for mRNA quantitation. Biotechniques 39:75-85

26. Asghari G, Jalali M, Sadoughi E (2012) Antimicrobial activity and chemical composition of essential oil from the seeds of Artemisia aucheri Boiss. Jundishapur journal of natural pharmaceutical products 7:11

27. Gharehmatrossian S, Popov YU, Ghorbanli M, Safaeian S (2012) Antioxidant activities and cytotoxic effects of whole plant and isolated culture of Artemisia aucheri Boiss. Asian Journal of Pharmaceutical Clinical Research 5:95-98

\section{Table 2}

Table 2 is not available with this version of the manuscript.

\section{Figures}

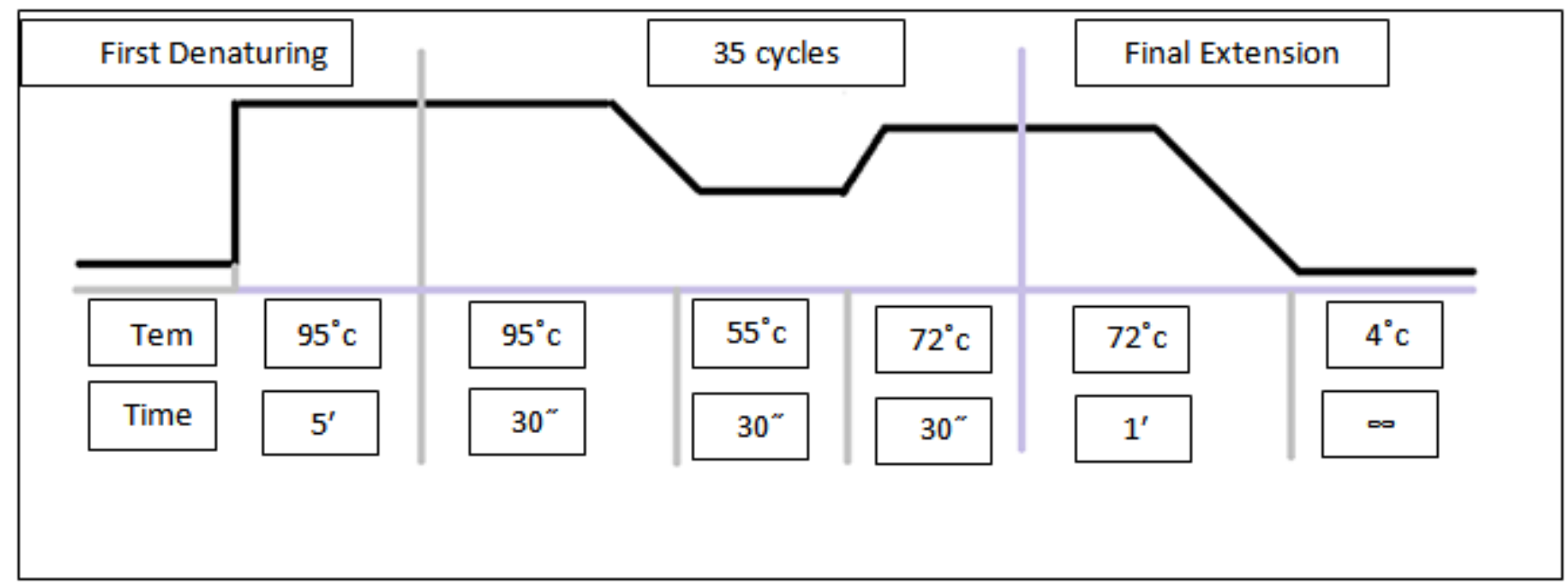

\section{Figure 1}

Thermocycler device for sequence amplification. 


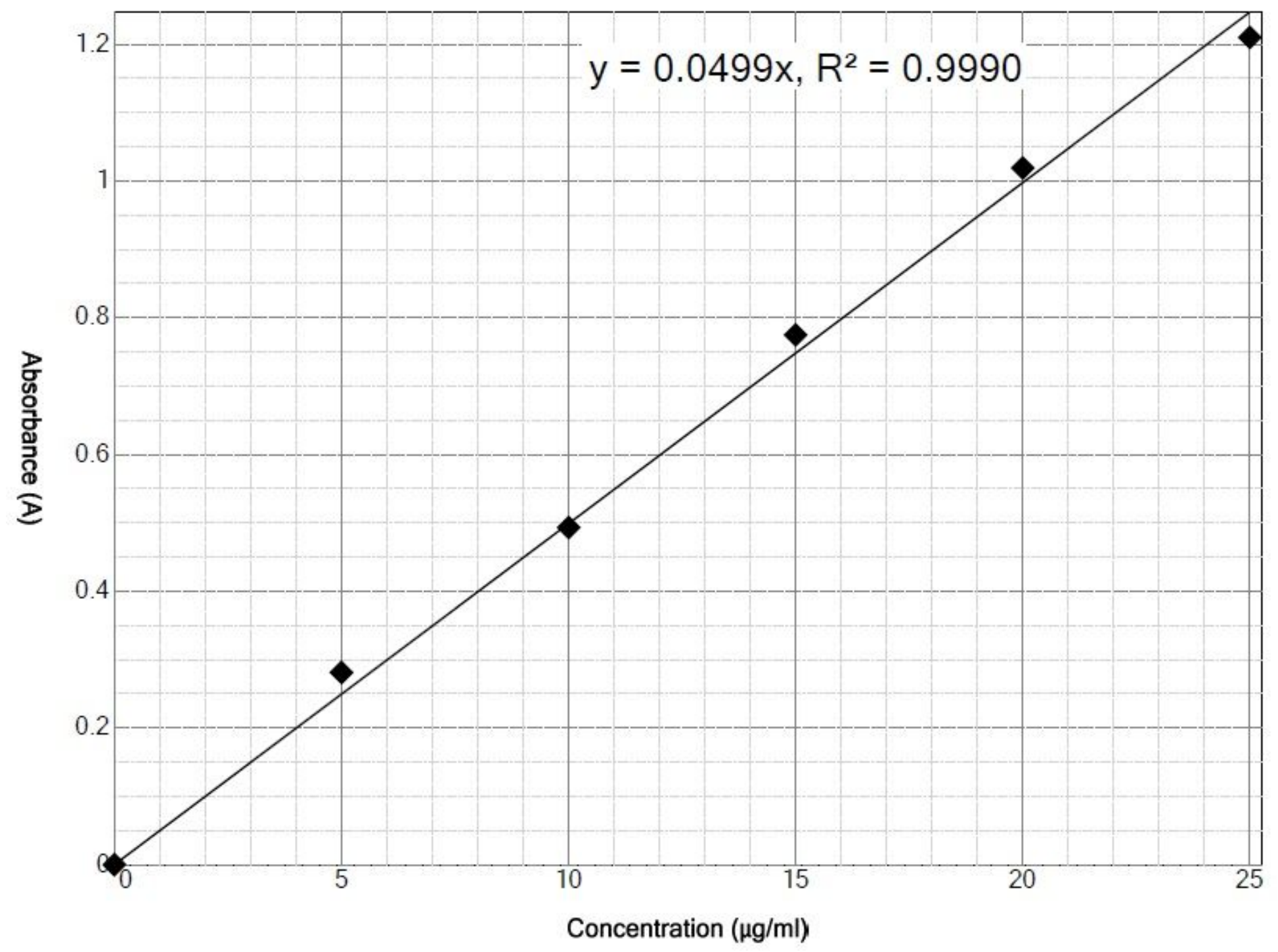

Figure 2

Standard curve of Artemisia extraction at $274 \mathrm{~nm}$ of wavelength. 
Size (d.n... $\quad \%$ Intensity: St Dev (d.n...

Z-Average (d.nm): 113.20

Peak 1: 104.5

98.0

75.33

Pdl: 0.216

Peak 2: $\quad 4244$

2.0

988.8

Intercept: 0.917

Peak 3: $\quad 0.000$

0.0

0.000

Result quality Good

Size Distribution by Intensity

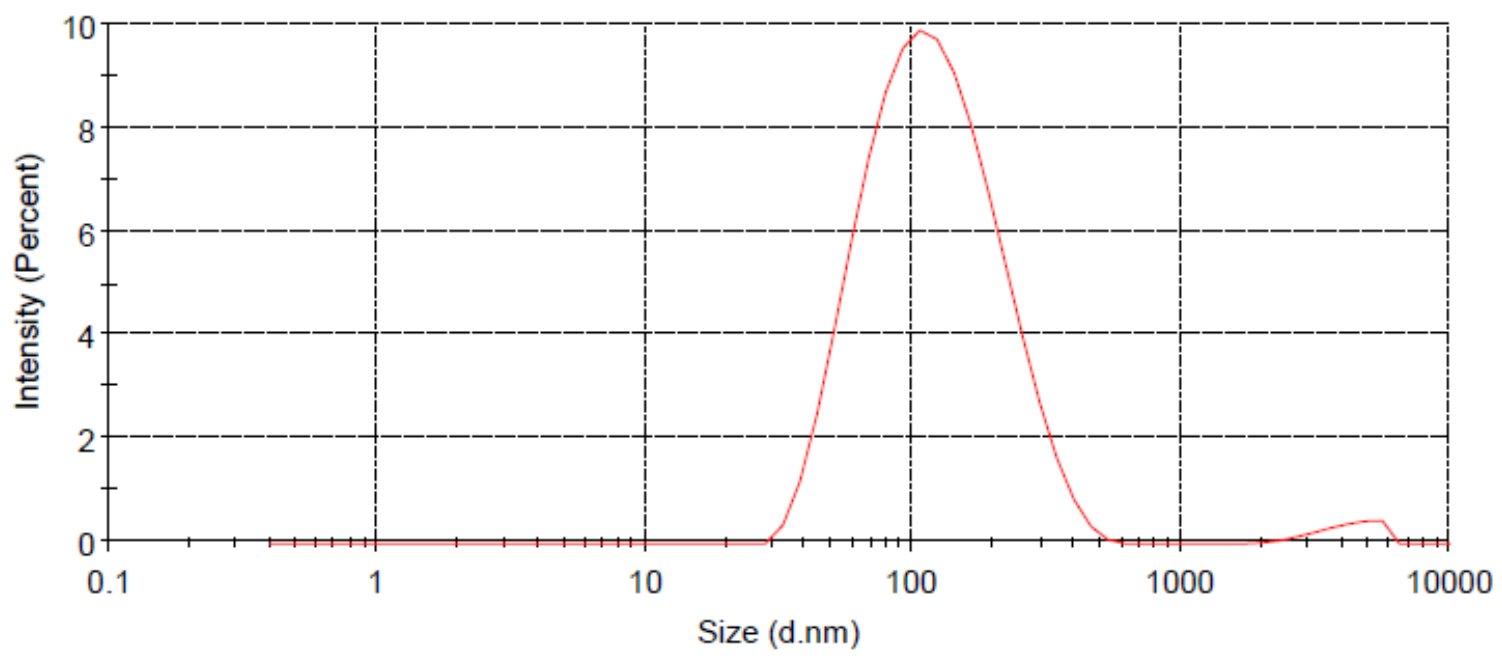

\section{Figure 3}

An example of the spectrum obtained from particle size determination and dispersion index in the ARSLN3 formulation. 


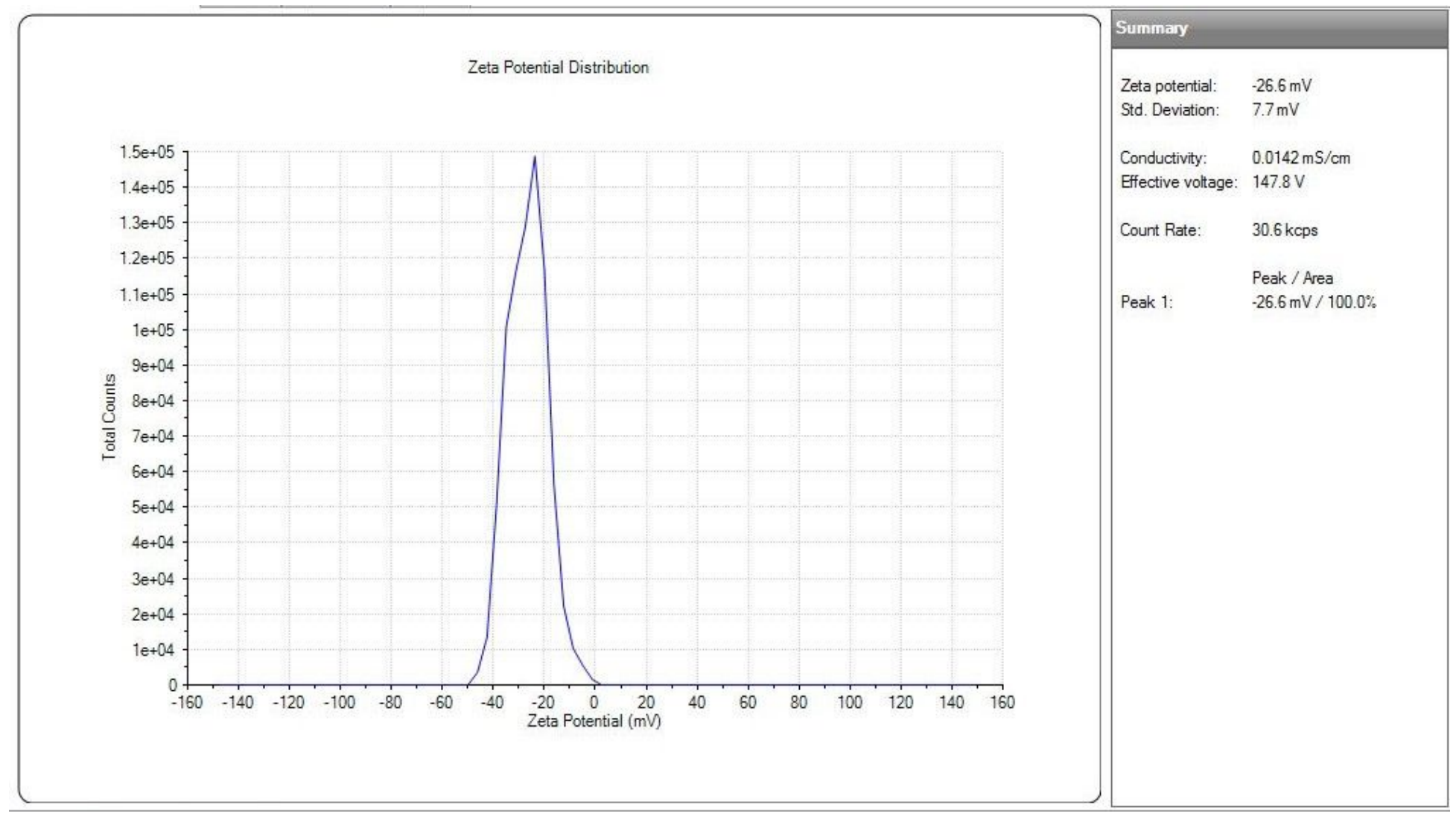

Figure 4

An example of zeta potential in the AR-SLN3 formulation. 
TEM image of SLN containing spherical Artemisia extraction.

\section{Release Profile}

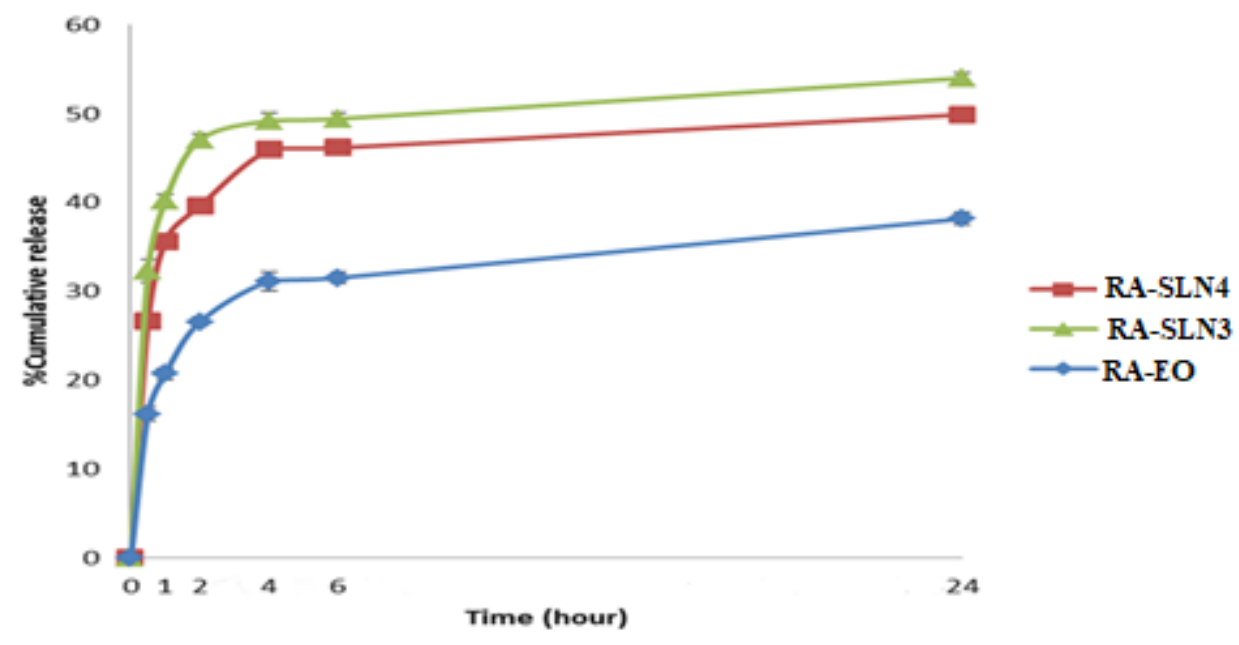

Figure 6

Comparative graph of control release, RA-SLN4 and RA-SLN3 formulation in buffer environment PH=7.4

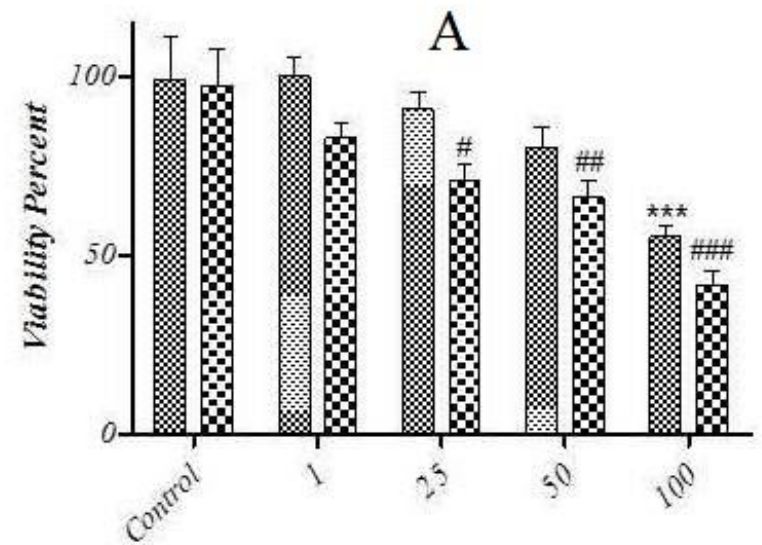

Concentration (mM)

$\mathrm{C}$

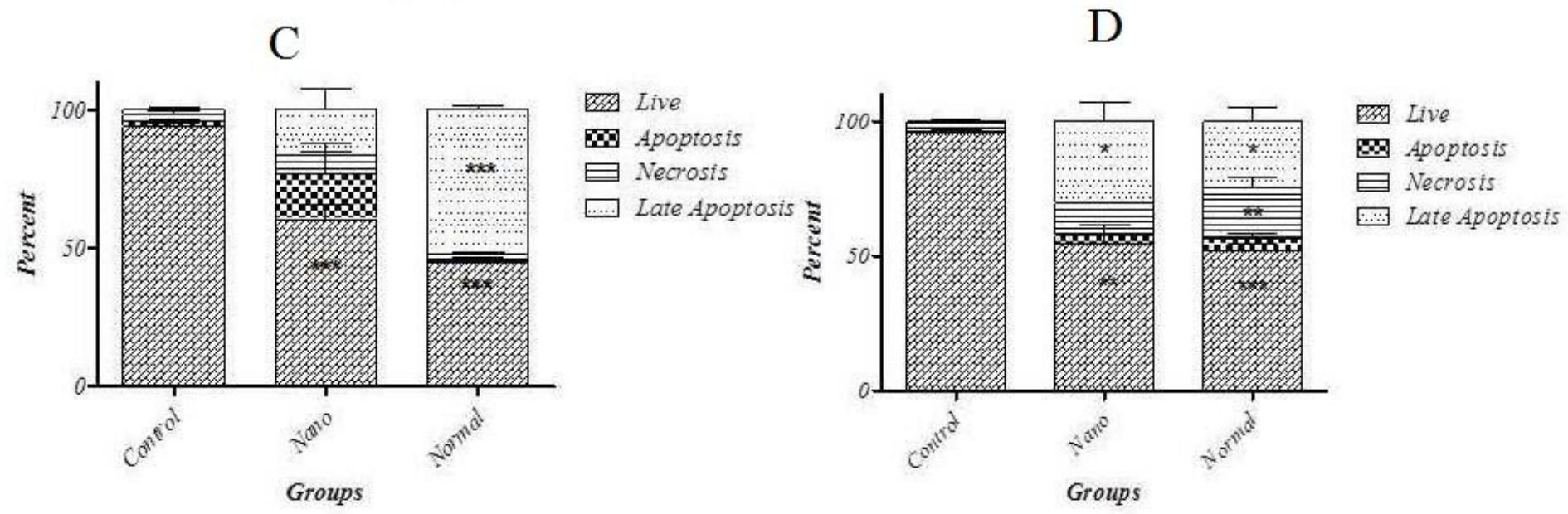

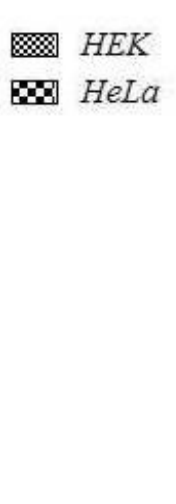

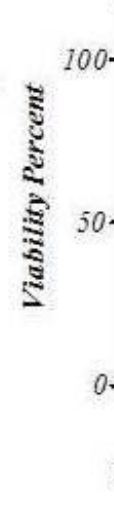

B

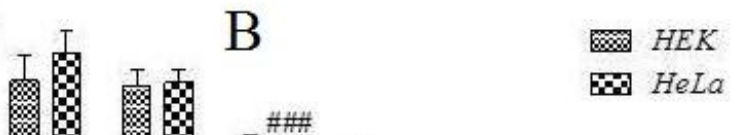

Figure 7 
Percentage of cell inhibition by Artemisia extraction (A), lipid nanoparticles containing extraction of mountain treatment plant (B), induction of Apoptosis by lipid nanoparticles containing extraction on HEK293 (C) and SKOV-3 (D).
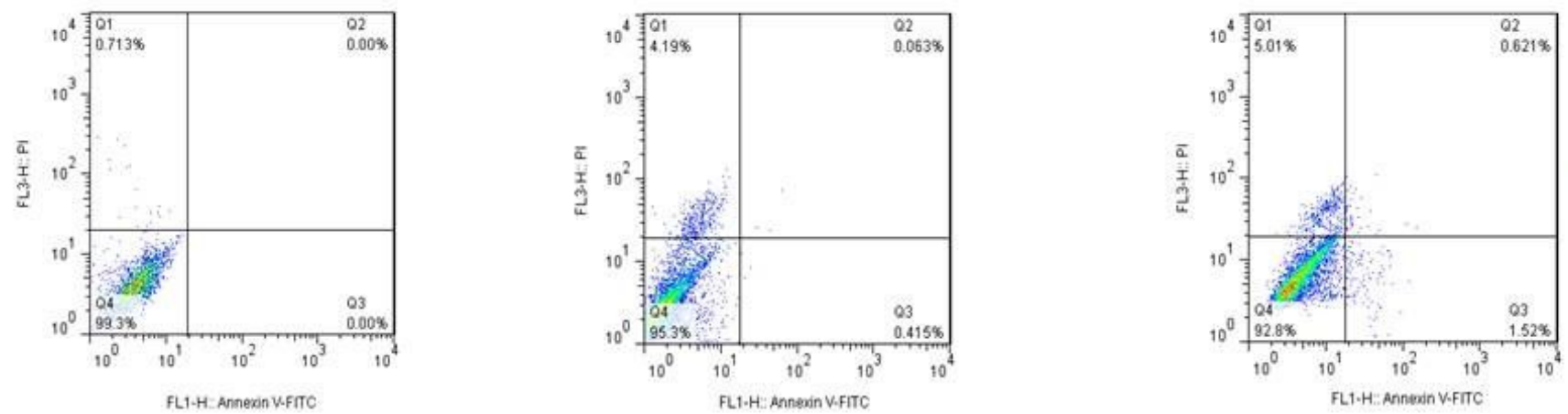

A
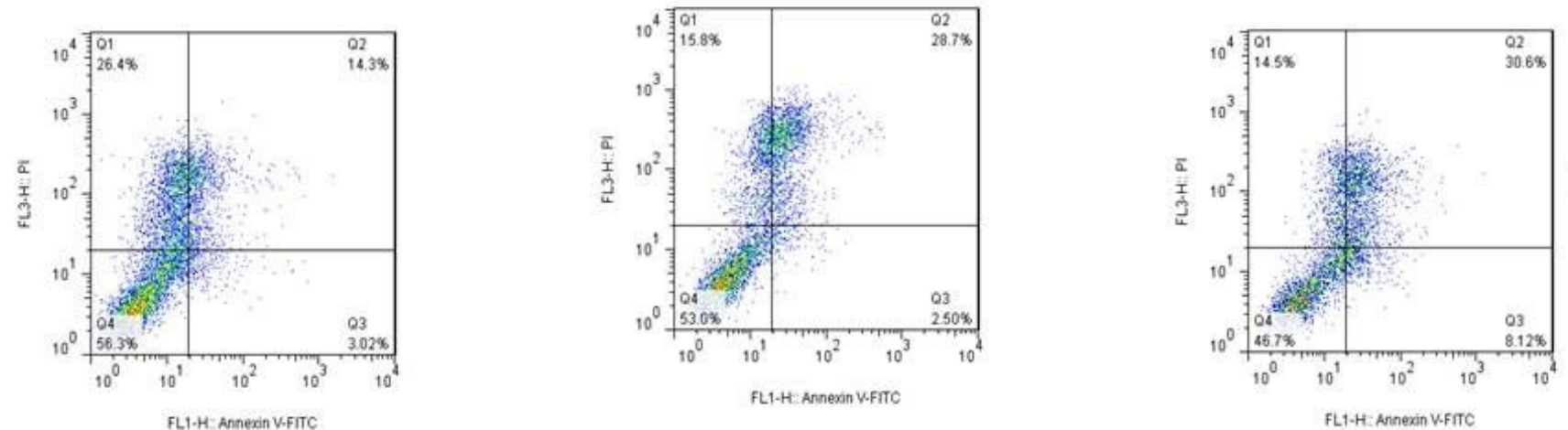

B
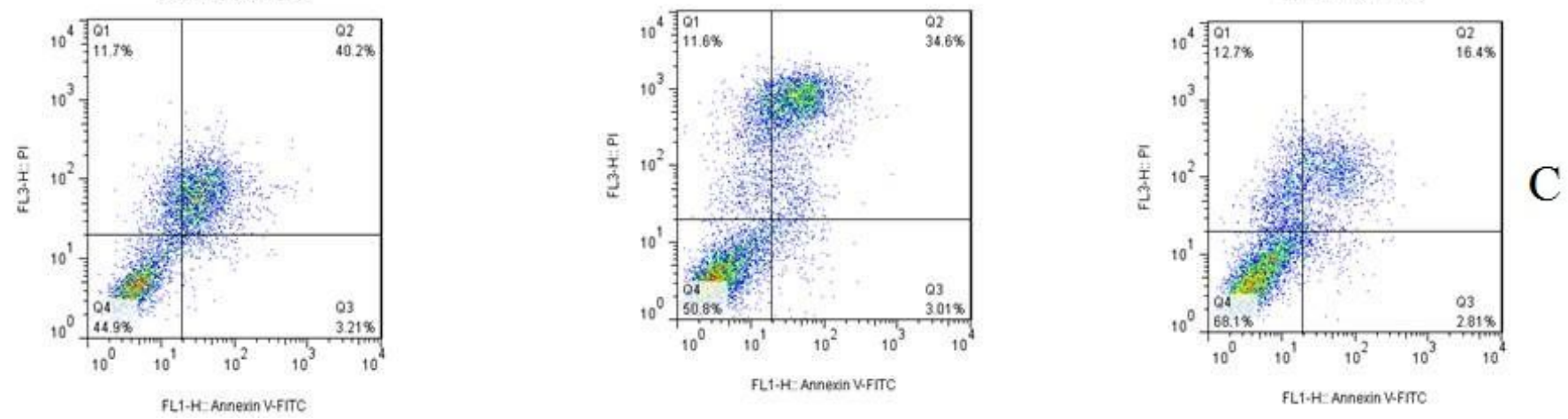

Figure 8

The rate of apoptosis in SKOV-3 cells under control conditions (A); under Artemisia extraction treatment (B); and under the treatment of nanoparticles containing Artemisia extraction (C). 

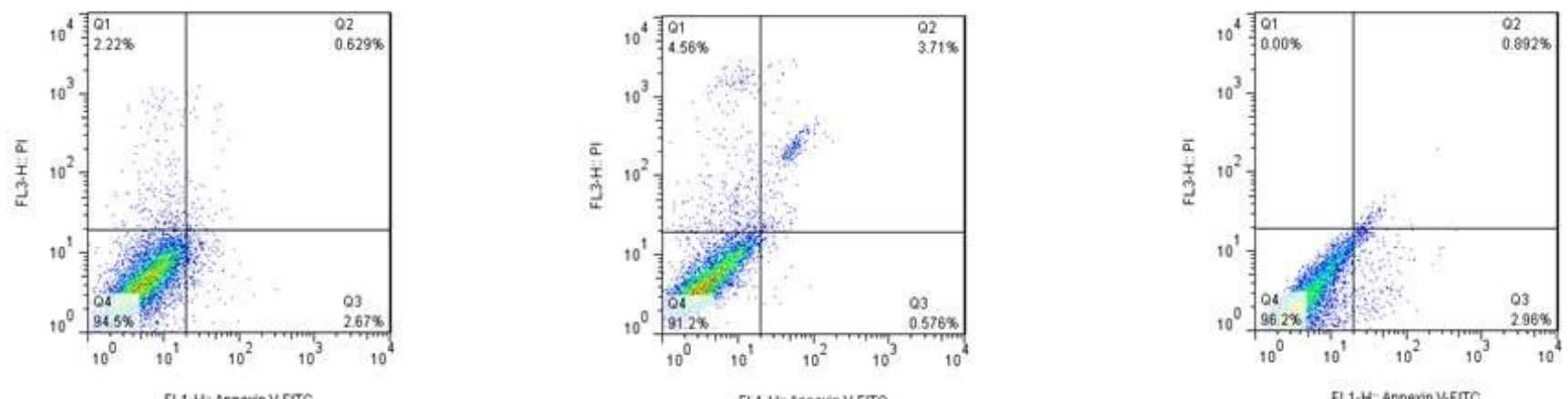

A
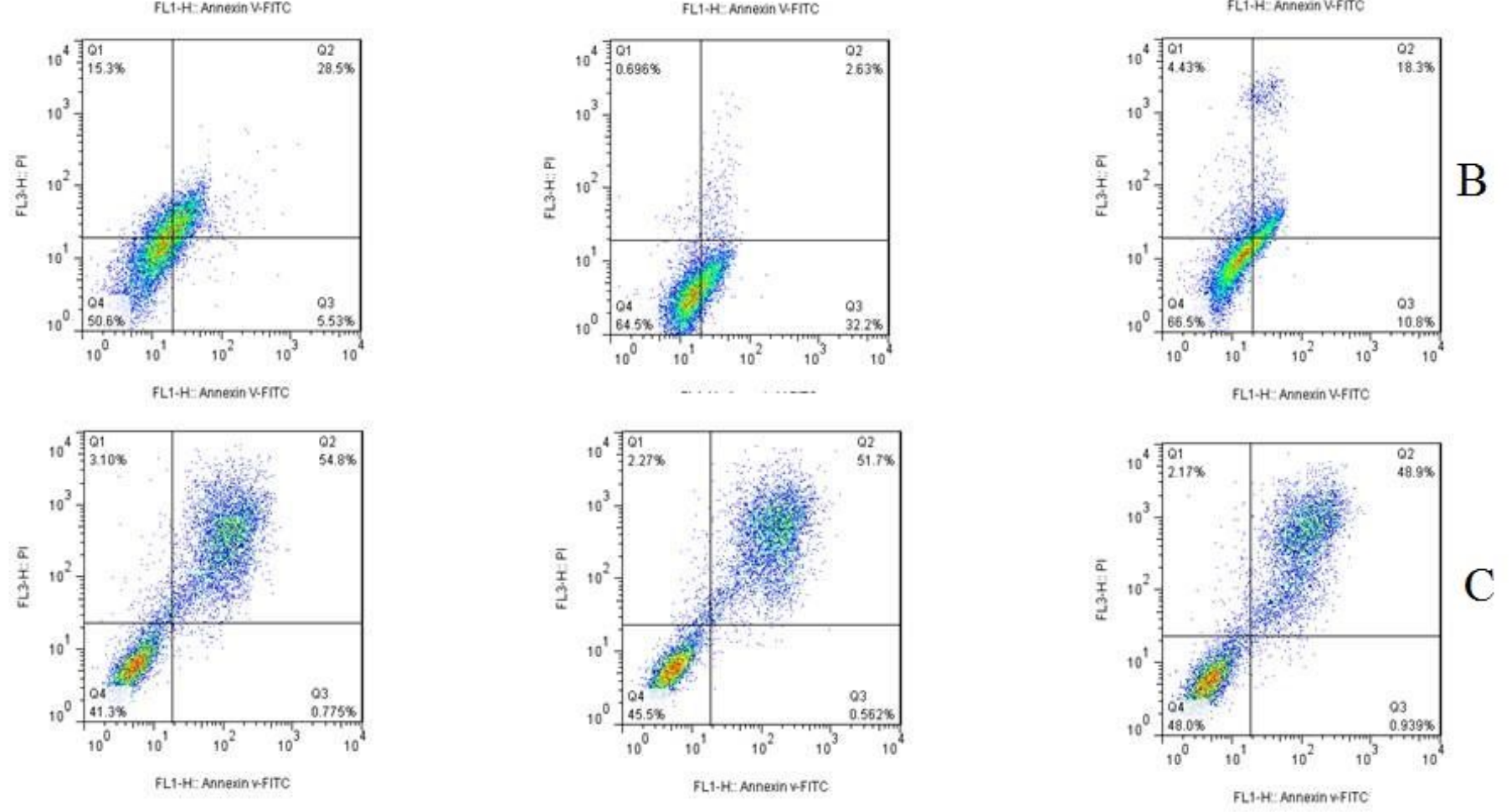

Figure 9

The rate of apoptosis in SKOV-3 cells under control conditions (A); under Artemisia extraction treatment (B); and under the treatment of nanoparticles containing Artemisia extraction (C). 

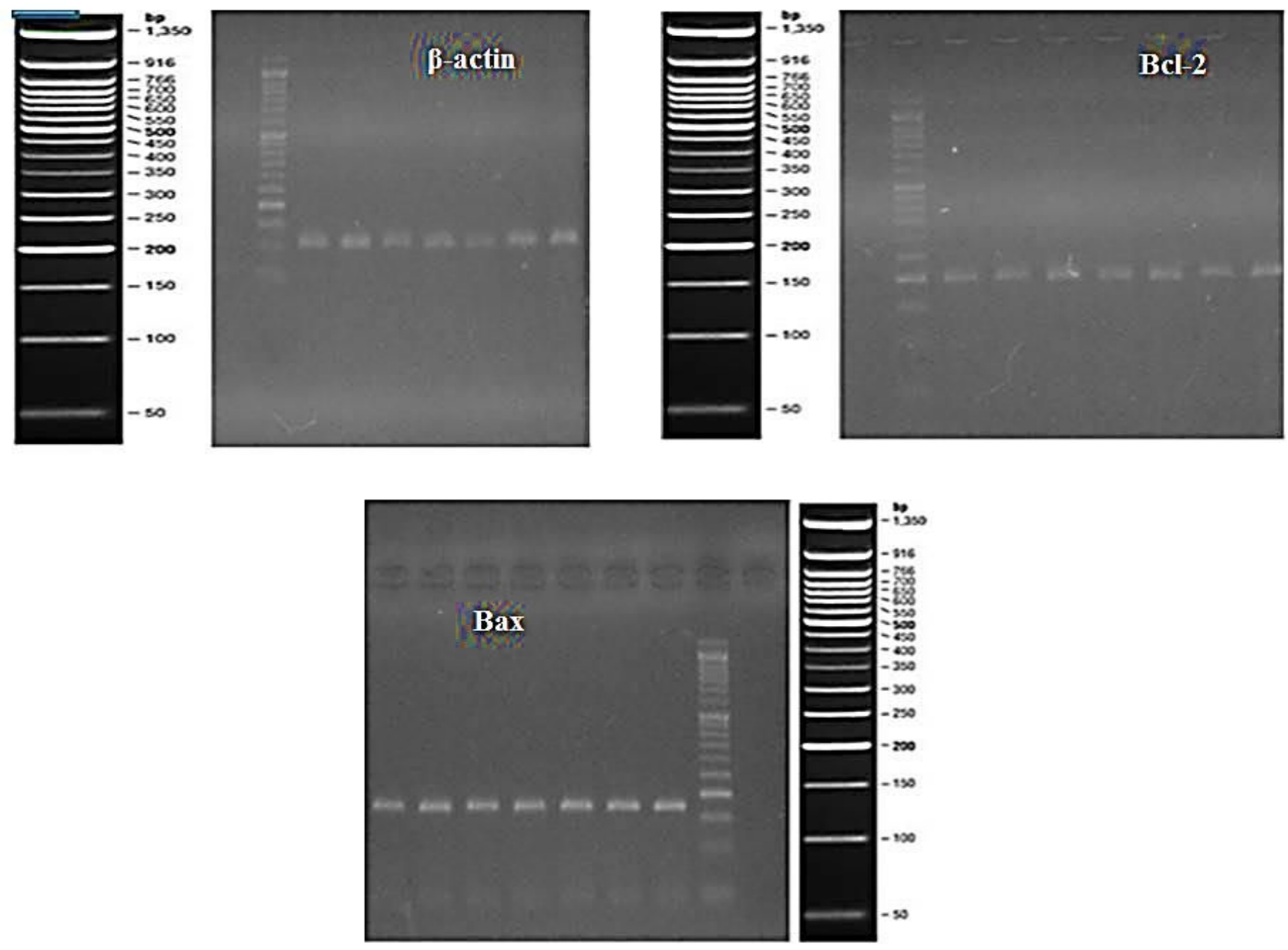

Figure 10

Quality of Cdna synthesized on agarose gel $2 \%$ from top to bottom BCL-2, Bax, and $\beta$-actin respectively. 

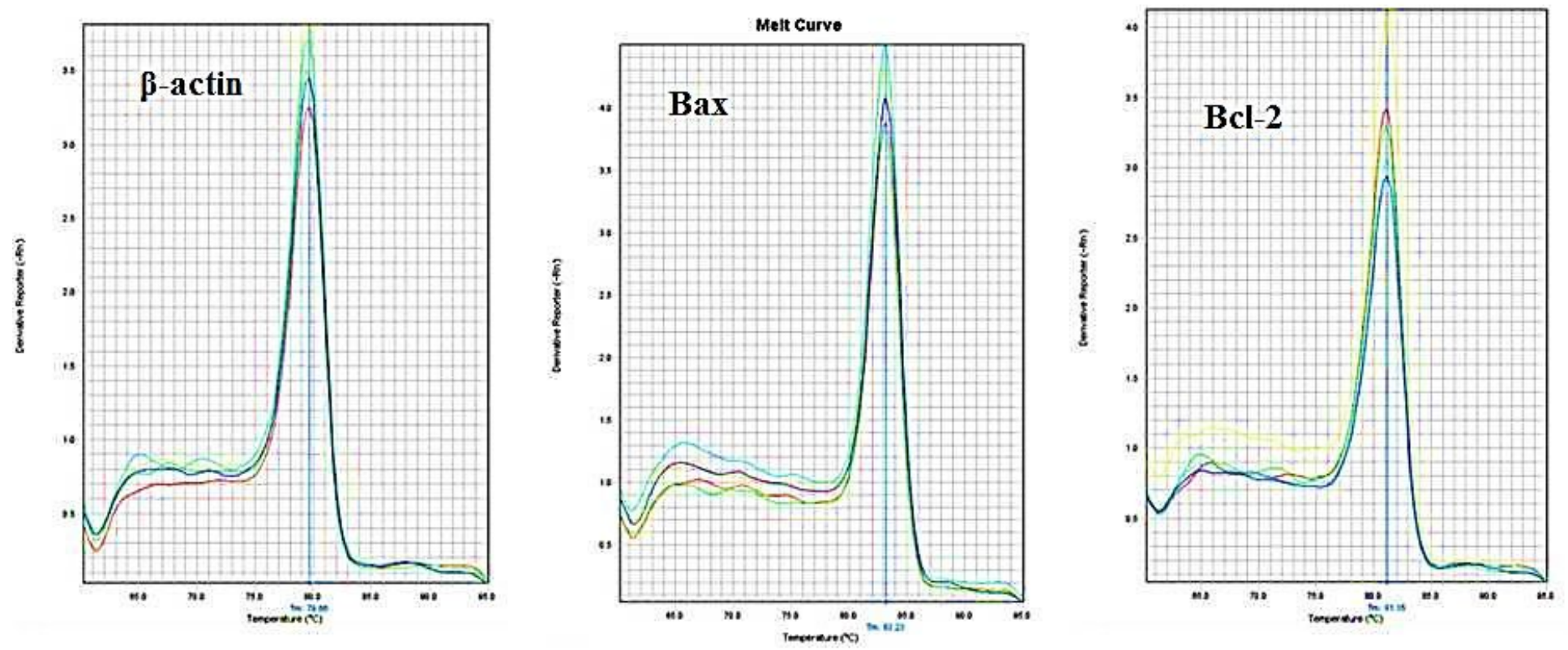

Figure 11

Melting curve for genes BCL-2, Bax, and $\beta$-actin 